\title{
Wash-free, antibody-assisted magnetoreduction assays of orchid viruses
}

\author{
S.Y. Yang ${ }^{\text {a }}$, Z.F. Jian ${ }^{\text {a }}$, J.J. Chieh ${ }^{\text {a }}$, H.E. Horng ${ }^{\mathrm{a}, *}$, H.C. Yang ${ }^{\mathrm{b}}$, \\ I.J. Huang ${ }^{\mathrm{c}}$, Chin-Yih Hong ${ }^{\mathrm{d}, * *}$ \\ ${ }^{a}$ Institute of Electro-optical Science and Technology, National Taiwan Normal University, Taipei 116, Taiwan \\ ${ }^{\mathrm{b}}$ Department of Physics, National Taiwan University, Taipei 106, Taiwan \\ ${ }^{\mathrm{c}}$ Taiwan Sugar Research Institute, Tainan 701, Taiwan \\ ${ }^{\mathrm{d}}$ Department of Mechanical Engineering, Nan-Kai Institute of Technology, Nantou County, Taiwan \\ Received 9 August 2007; received in revised form 14 January 2008; accepted 16 January 2008 \\ Available online 25 March 2008
}

\begin{abstract}
This study demonstrates the feasibility of wash-free magnetoreduction assays (MRA) of orchid viruses. A magnetic reagent, consisting of magnetic beads coated with antibodies and dispersed in water, was synthesized. By using a mixed-frequency alternative-current (ac) magnetosusceptometer, differences in the magnetic susceptibilities of the magnetic reagent before and after the addition of orchid solutions were measured. The results show significant advantages for MRA of orchid viruses.
\end{abstract}

(C) 2008 Elsevier B.V. All rights reserved.

Keywords: Magnetoreduction; Magnetic beads; Orchid virus; Assay

Assays of plant viruses have always been important in agriculture. The existence of a variety of efficient and convenient assay methods has helped to enhance the economic benefit of plants and has allowed the monitoring of the spread of plant viruses. At present, there are several types of assays for plant viruses, such as direct microscopy (Francki and Grivell, 1970; Hibino et al., 1974), polymerase chain reactions (PCR) (Ryu and Park, 1995; Teycheney et al., 2007), and enzymelink immunosorbent assay (ELISA) (Vejaratpimol et al., 1998; Leyva et al., 2007), and others. Although these techniques have been applied widely, weaknesses of these techniques have been discovered in practical trials. For instance, complicated processes and expensive instruments are involved in microscope-observing assays and PCR, which reduces greatly the through-put of samples. For ELISA, two types of antibodies are required simultaneously, for coating and for marking a given virus and the assay also involves tedious assay processes. These disadvantages may cause failures in assaying certain viruses when only one type of antibody is available. Such flaws motivate

\footnotetext{
* Corresponding author. Tel.: +886 229338260; fax: +886 286631954 .

** Corresponding author.

E-mail addresses: phyfv001@scc.ntnu.edu.tw (H.E. Horng), cyhong@nkc.edu.tw (C.-Y. Hong).
}

the search for alternative forms of easy, efficient, and versatile assays for plant viruses.

In a previous study (Hong et al., 2006), a technology involving the measurements of reduction in the ac magnetic susceptibility of a magnetic reagent was proposed. This technique, referred as magnetoreduction assay (MRA), was used to assay human proteins (e.g. c-reactive protein) in serum (Hong et al., 2007). MRA uses magnetic beads coated with one type of antibody as markers for the to-be-detected biomolecules (Jiang et al., 2004). Under the actions of multiple alternativecurrent (ac) magnetic fields, individual magnetic nanoparticles dispersed in aqueous solution rotate in response to applied ac magnetic fields. Since each magnetic bead possesses magnetic moment, the reagent exhibits ac magnetic susceptibility $\chi_{\text {ac }}$ under external ac magnetic fields. When a sample containing the to-be-detected biomolecules is mixed with a magnetic reagent, magnetic nanoparticles in the reagent associate with the to-be-detected biomolecules via the formation of immune complexes of biomolecules-antibodies-magnetic-beads, as shown schematically in Fig. 1. Because they are confined physically, the clustered magnetic nanoparticles cannot rotate in response to the applied ac magnetic fields, reducing the ac magnetic susceptibility of the magnetic reagent. Therefore, to-be-detected biomolecules in samples can be assayed by measuring the reduction in ac magnetic susceptibility of the magnetic 


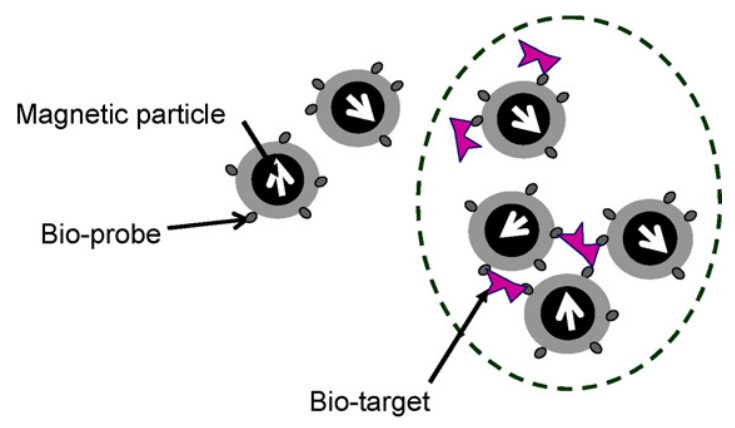

Fig. 1. Scheme for the association between the to-be-detected biomolecules and magnetic beads coated with antibodies. The magnetic beads become larger or clustered due to binding with to-be-detected biomolecules, as circled with the dashed line.

reagent following the addition of samples into the magnetic reagents.

As can be seen from this description, MRA has several unique merits. First, unbound to-be-detected biomolecules and magnetic beads do not need to be removed, because they are allowed in the reagent. As a result, the assay process of MRA is comparatively simple. Second, only one kind of antibody is used in MRA. Third, MRA is a direct and homogeneous assay, which is a type of assay that usually has a high degree of reliability and sensitivity. Fourth, because the amount of reduction in $\chi_{\mathrm{ac}}$ can be measured accurately to correspond to the concentration of the to-be-detected biomolecules, the concentration of biomolecules can thus be measured quantitatively. With such merits for practical trials, MRA is a promising candidate for efficiently and conveniently detecting plant viruses. In this work, a platform that is based on MRA to assay plant viruses is developed. The advantages of MRA in this context are demonstrated by assaying two orchid viruses, odontoglossum ringspot virus (ORSV) and cymbidium mosaic virus (CyMV).

Magnetic beads were dispersed in phosphate buffer saline (PBS) solution and were coated with dextran (MagQu Co.) over a core material of $\mathrm{Fe}_{3} \mathrm{O}_{4}$. By using a magnetic force microscope (MFM), the topologies of the hundreds of magnetic beads were probed. With these MFM images, the mean value and the standard deviation in diameter of the magnetic beads were found to be $29.3 \mathrm{~nm}$ and $1.4 \mathrm{~nm}$, respectively.

To magnetically label ORSV or CyMV, anti-ORSV or antiCyMV (Dr. Chip Biotechnology Inc.) was coated onto dextran enveloping magnetic cores. To bind anti-ORSV (or anti-CyMV) to dextran, dextran was oxidized to create aldehyde groups (-CHO) onto the dextran. Then it reacted to the anti-ORSV (or anti-CyMV) solution with aldehyde groups of dextran to form $-\mathrm{CH}=\mathrm{N}-$. Through magnetic separation, unbound antiORSV (or anti-CyMV) was separated from the solution. Reagent with anti-ORSV (or anti-CyMV) coated magnetic beads was obtained. Hereafter, the fluid containing anti-ORSV-dextran coated magnetic beads is denoted as ORSV magnetic reagent, and that containing anti-CyMV-dextran magnetic beads as CyMV magnetic reagent.

The mixed-frequency ac magnetic susceptibility $\chi_{\mathrm{ac}, \mathrm{o}}$ of a 50- $\mu$ l ORSV/CyMV magnetic reagent was measured with an ac magnetosusceptometer (MagQu Co.). Then 50- $\mu$ l mag- netic reagent was mixed with $50-\mu l$ orchid solution. After an 1.5-h incubation period for the formation of immune complexes of ORSV-anti-ORSV-dextran-coated-magnetic-beads (or CyMV-anti-CyMV-dextran-coated-magnetic-beads) at room temperature, the mixed-frequency ac magnetic susceptibility for the mixture of 50- $\mu$ l magnetic reagent and 50- $\mu$ l orchid solution was measured (referred to as $\chi_{\mathrm{ac}}^{\prime}$ ). The $\chi_{\mathrm{ac}}^{\prime}$ was expected to be less than $\chi_{\mathrm{ac}, \mathrm{o}}$ whenever immune complexes were formed. It is worthy of note that the entire process for assaying an orchid virus via MRA is relatively simple: mix the reagent and the sample, incubate the result for one and a half hours at room temperature, and finally measure the reduction in $\chi_{\mathrm{ac}}$ to determine the existence of the orchid virus. Compared to the process of ELISA, which usually needs two cycles of incubation and involves wash/separation processes, it is clear that MRA has a number of advantages over ELISA. For example, MRA involves less time and has wash-free operations. In addition, because the antibodies used in MRA may be just half of that required in ELISA, the cost of reagents, and thus the cost for each test, is less than that of ELISA. Furthermore, the signal detected in MRA is magnetic, instead of the optical signal in ELISA, so the dependence of the signal on sample color is reduced greatly for MRA.

To examine the coating of anti-ORSV (or anti-CyMV) onto dextran on magnetic beads, anti-IgG-HRP (Abcom Inc.) was added into the ORSV/CyMV magnetic reagents to be bound to the anti-ORSV (or anti-CyMV). Through incubation and magnetic separation, unbound anti-IgG-HRP was removed from the magnetic reagents. Then we detected the optical density emitted by the HRP bound to the anti-ORSV (or anti-CyMV) in the magnetic reagents via ELISA. For comparison, fluid containing dextran-coated magnetic beads that was not coated with antiORSV (or anti-CyMV) was treated by adding anti-IgG-HRP, incubation, magnetic separation, and ELISA. The results are shown in Fig. 2(a) and (b), which show the results of examinations of the coating of anti-ORSV and anti-CyMV onto dextran, respectively.

In Fig. 2(a), the optical density for the ORSV magnetic reagent was measured to be $1.38 \pm 0.12$. The background, which corresponds to the measured optical density of the dextran-coated magnetic beads that were not coated with antiORSV/anti-CyMV, was found to be $0.71 \pm 0.14$. The clear increase in the optical density of the ORSV magnetic reagent, as compared to the background in Fig. 2(a), reveals that the dextran-coated magnetic beads were labeled with HRP via the linker of anti-ORSV-anti-IgG-HRP (or anti-CyMV-anti-IgGHRP). Thus, anti-ORSV was definitely coated onto the dextran on the magnetic beads. The significant increase in optical density of the CyMV magnetic reagent, in as compared to the background was also observed and shown in Fig. 2(b). Therefore, anti-ORSV and anti-CyMV were definitely bound onto the magnetic beads.

To investigate the validity of MRA in the orchid viruses ORSV and CyMV, three orchid solutions (denoted as S1, S2, and S3) were used as detected samples. According to the results from PCR and ELISA, S1 was infected with ORSV, S2 was infected with CyMV, and S3 was infected with neither ORSV 

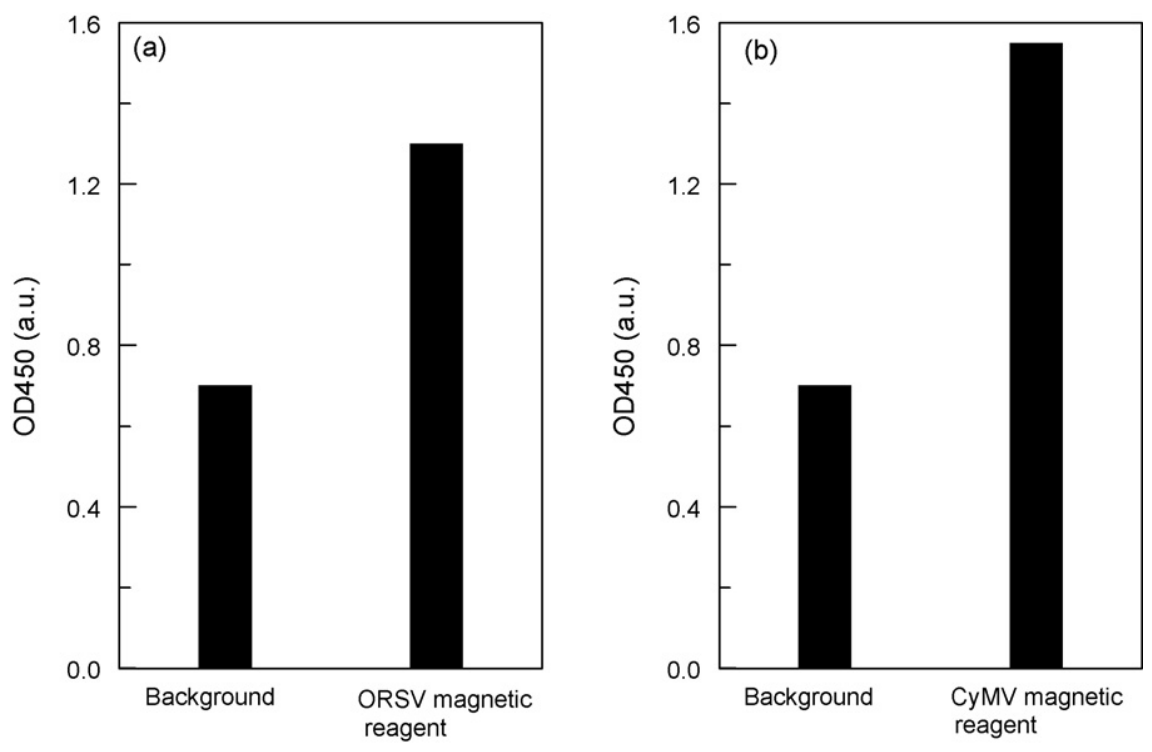

Fig. 2. Comparisons of ELISA signals OD450 between (a) magnetic fluid and ORSV magnetic reagent, and (b) magnetic fluid and CyMV reagent.

nor CyMV. It is note worthy that the samples with ORSV or CyMV of known concentrations are not easily available. In this work, the results of PCR and ELISA are only qualitatively meaningful.

When ORSV magnetic reagent was mixed with each of these samples, measured the reduction in the mixed-frequency ac magnetic susceptibility $\left(\Delta \chi_{\mathrm{ac}}=\chi_{\mathrm{ac}, \mathrm{o}}-\chi_{\mathrm{ac}}^{\prime}\right)$ of the mixtures shows that the S1 solution were $0.97 \pm 0.04 \%$ for $\Delta \chi_{\mathrm{ac}} / \chi_{\mathrm{ac}, 0}$, and the S2 and S3 solutions showed $0.03 \pm 0.03 \%$ and $0.02 \pm 0.02 \%$ for $\Delta \chi_{\mathrm{ac}} / \chi_{\mathrm{ac}, \mathrm{o}}$, respectively, as shown in Fig. 3(a). This implies that some anti-ORSV-dextran-coated magnetic beads had become clustered in the S1 solution, whereas the magnetic beads did not cluster in the solutions S2 and S3. The MRA results shown

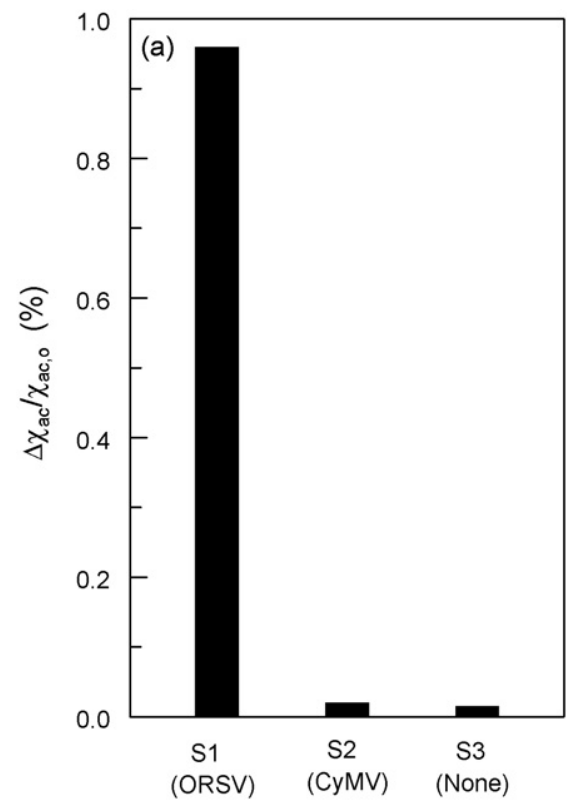

Fig. 3(a) indicate that only the S1 solution was infected with ORSV.

The results regarding the MRA on CyMV for these samples are shown in Fig. 3(b). CyMV magnetic reagent was mixed with each of the samples. As can be seen from Fig. 3(b), solution S2 had a significant reduction in its mixed-frequency ac magnetic susceptibility $\left(\Delta \chi_{\mathrm{ac}} / \chi_{\mathrm{ac}, \mathrm{o}}=0.61 \pm 0.07 \%\right)$, whereas the values of $\Delta \chi_{\mathrm{ac}} / \chi_{\mathrm{ac}, \mathrm{o}}$ for solutions $\mathrm{S} 1$ and S3 were almost zero. Thus, the MRA for the CyMV indicates that only the S2 solution was infected with CyMV. Therefore, the MRA results shown in Fig. 3(a) and (b) are consistent with those via PCR and ELISA.

In conclusion, the viruses ORSV and CyMV in orchids were detected with the synthesis of a magnetic reagent and

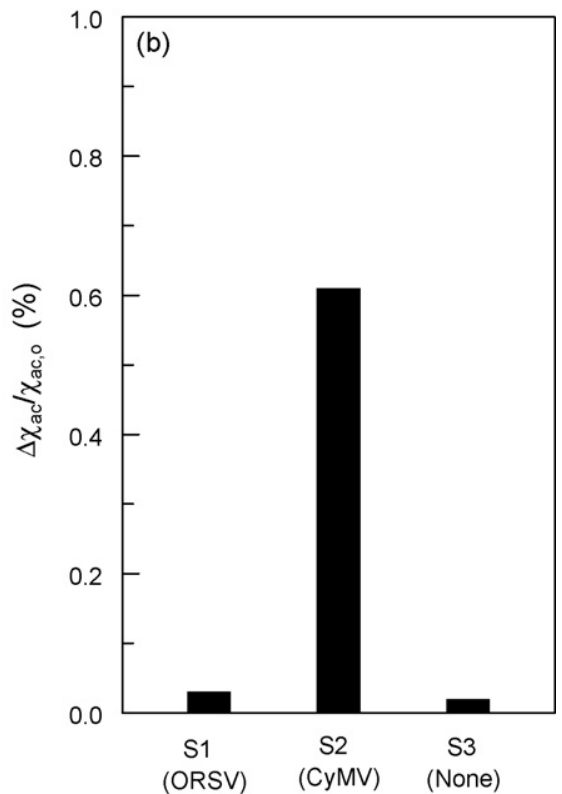

Fig. 3. Magnetoreduction assaying signals $\Delta \chi_{\mathrm{ac}} / \chi_{\mathrm{ac}, \mathrm{o}}$ of three orchid samples using (a) ORSV magnetic reagent, and (b) CyMV magnetic reagent. These first two orchid samples were infected with ORSV and CyMV, respectively. The third sample was not infected with ORSV and CyMV. 
using a mixed-frequency ac magnetosusceptometer. The magnetoreduction assays (MRAs) for orchid viruses ORSV and CyMV demonstrated a number of advantages, including washfree operations, shorter operation time, lower-cost reagent, and sample-color independence. It is believed that MRA is very promising for use in practical trials to assay orchid viruses.

\section{Acknowledgements}

This work was supported by the National Science Council of Taiwan under Grant Nos. 95-2120-M-003-001, 95-2112-M-003-017-MY2, 96-2112-M-002-025, 96-2752-M002-016-PAE, and 96-2628-M-003-002.

\section{References}

Francki, R.I.B., Grivell, C.J., 1970. An electron microscope study of the distribution of tomato spotted wilt virus in systemically infected Datura stramonium leaves. Virology 42, 969-978.

Hibino, H., Tsuchizaki, T., Saito, Y., 1974. Comparative electron microscopy of cytoplasmic inclusions induced by 9 isolates of soil-borne wheat mosaic virus. Virology 57, 510-521.
Hong, C.-Y., Chen, W.H., Chien, C.F., Yang, S.Y., Horng, H.E., Yang, L.C., Yang, H.C., 2007. Wash-free immunomagnetic detection for serum through magnetic susceptibility reduction. Appl. Phys. Lett. 90, 74105.

Hong, C.-Y., Wu, C.C., Chiu, Y.C., Yang, S.Y., Horng, H.E., Yang, H.C., 2006. Magnetic susceptibility reduction method for magnetically labeled immunoassay. Appl. Phys. Lett. 88, 212512.

Jiang, W.Q., Yang, H.C., Yang, S.Y., Horng, H.E., Hung, J.C., Chen, Y.C., Hong, C.-Y., 2004. Preparation and properties of superparamagnetic nanoparticles with narrow size distribution and biocompatible. J. Magn. Magn. Mater. 283, 210-212.

Leyva, A., Franco, A., González, T., Sánchez, J.C., López, I., Geada, D., Hernández, N., Montañés, M., Delgado, I., Valdés, R., 2007. A rapid and sensitive ELISA to quantify an HBsAg specific monoclonal antibody and a plant-derived antibody during their downstream purification process. Biologicals 35, 19-25.

Ryu, K.H., Park, W.M., 1995. Rapid detection and identification of odontoglossum ringspot virus by polymerase chain reaction amplification. FEMS Microbiol. Lett. 133, 265-269.

Teycheney, P.-Y., Acina, I., Lockhart, B.E.L., Candresse, T., 2007. Detection of Banana mild mosaic virus and Banana virus $\mathrm{X}$ by polyvalent degenerate oligonucleotide RT-PCR (PDO-RT-PCR). J. Virol. Meth. 142, 41-49.

Vejaratpimol, R., Channuntapipat, C., Liewsaree, P., Pewnim, T., Ito, K., Iizuka, M., Minamiura, N., 1998. Evaluation of enzyme-linked immunosorbent assays for the detection of cymbidium mosaic virus in orchids. J. Ferment. Bioeng. 86, 65-71. 